\title{
A Disassembly Model based on Polychromatic Sets Theory for Manufacturing Systems
}

\author{
Lijun Yan', Lili Jiang ${ }^{2}$, and Zongbin $\mathrm{Li}^{1}$ \\ 1 State Key laboratory for Manufacturing Systems Engineering, Xi'an \\ Jiaotong University, Xi'an, 710049, China. \\ yljylp@yahoo.com.cn, lzb9910@mail.xjtu.edu.cn \\ WWW home page: http://www.xjtu.edu.cn/en/academics\&research/me.htm \\ 2 CALS Research Center, Guangdong technical University, Guangdong \\ 510090 , China. Jiang_l18888@126.com \\ WWW home page: http://www.gdut.edu.cn/
}

\begin{abstract}
Existing disassembly models are inefficient in generation of disassembly sequence and the number of disassembly schemes tends to be explosive in combination. In addition, few models have focused on threedimension disassembly. This paper defines the concept of connectivity of part unit, proposes the generation algorithms of connective part units, and then develops a new disassembly model based on polychromatic sets theory. Adopting possible displacement model in polychromatic sets theory as disassembling model, all connective part units are sieved by possible displacement function groups to eliminate illogical ones. Hierarchical graph is employed to describe disassembly process and disassembly sequence. Present disassembly model based on polychromatic sets is convenient for expressing restriction relation between each two parts in disassembly process. It also has advantages of higher formation level, avoiding "combination explosion" and tending to program.
\end{abstract}

\section{Introduction}

Disassembly is a very important and difficult process in green remanufacturing engineering. The premise of recycling and reusing of worn-out products is that the 
parts of products that have arrived at their lifecycle and need recycling can be easily taken down. So before disassembling of products, disassembly process should be programmed properly and be optimized with the aims such as economics of disassembling, coordinating of environment and feasibility of technique. Finally optimal disassembly sequences can be obtained $[1,2]$.

In the literature there are many papers proposing various methods for studying disassembly and recycling. Moore (1998) proposed an algorithm which automatically generates a disassembly Petri Net (DDN) [3]. From a geometrically based precedence matrix, Gungor (1998) addressed the uncertainty related difficulties in disassembly sequence planning [4]. Then Lambert (2002) studied disassembly sequence generation for electric equipments [5]. Kongar (2002) presented a genetic algorithm for disassembly process planning [6]. Park (2003) proposed that a methodology generates an optimal disassembly sequence by determining disassembly methods, employing PLM (profit-loss margin) curve to determine disassembly depth, and using MRFD (modified reverse fishbone diagram) to generate optimal disassembly sequence [7]. Gupta (2004) studied disassembly problem based on a case of cell phone [8]. Gonzalez (2004) presented a scatter search (SS), in which metaheuristic is presented aiming to deal with the optimum disassembly sequence problem for the case of complex products with sequencedependent disassembly costs [9]. Chung (2004) presented an integrated approach to selective-disassembly sequence planning [10]. Villalba (2004) employed a recyclability index of material to determine economic of disassembly [11].

However, although many approaches to generate disassembly sequence have been developed in above literature, they have some common disadvantages as follows. They can not sieve illogical disassembly sequence, the number of disassembly schemes tends to be explosive in combination, it is difficult for them to differentiate different relations and to generate disassembly sequence, and few researches have focus on three-dimension disassembly [12].

\section{Connectivity of Part Units}

Connective part units are the really existent units in disassembly process which are composed of less than two parts. Their importance lies in their real existence in disassembly process. Connectivity of part units is based on the adjacent relation of parts which means that if two parts of the product have interface, they are adjacent [13]. To get generation algorithms of connective part units, firstly several definitions are made as follows.

Defination 1: If there are $\mathrm{n}$ parts in a product, then k-rank sub-graph of adjacent graph of the product can be formalized as Boolean vector $X^{k}=\left(X_{i}\right)$, where if part i belongs to the sub-graph, then $X_{i}=1$. Or $X_{i}=0$. Call $X^{k}$ k-rank Boolean vector of adjacent graph and it is denoted as $\left\|X^{k}\right\|=k$. All k-rank Boolean vectors of 
adjacent graph compose k-rank Boolean vector set which is noted as $X^{(k)}$. $X^{(k)}=\left\{X^{k}\left\|X^{k}\right\|=k\right\}$.

Defination 2: With $X_{1}$ and $X_{2}$ as base, defining binary relation as follows:

$R=\left\{\left\langle x_{1}, x_{2}\right\rangle \mid\left\|x_{1} \oplus x_{2}\right\|_{1}=k, x_{1} \in X_{1}, x_{2} \in X_{2}\right\}$, so there exist that $\mathrm{Y}$ $\left(Y \in Y^{k}\right), Y=\left\{x_{1} \oplus x_{2} \mid\left\langle x_{1}, x_{2}\right\rangle \in R\right\}$ and call Y k-rank combinatorial Boolean vector of $X_{1}$ and $X_{2} . Y$ is denoted as $Y=\left.\left(x_{1}, x_{2}\right)\right|_{\oplus} ^{k}$.

Reasoning 1: provided that It has been known that there are $\mathrm{n}$ parts in some product, its adjacent matrix is $A_{p}=\left(a_{i j}\right)$. If $a_{i j}=1(1 \leq i<j \leq n)$, then $S^{2}=\left(S_{t}\right)$ can be constructed, Where there is only $S_{i}=S_{j}=1 . S^{(2)}$ may be constructed using $S^{2}$ as elements.

Reasoning 2: If $S^{(k-1)}$ is known, then $S^{(k)}=\left.\left(S^{(k-1)}, S^{(k-1)}\right)\right|_{\oplus} ^{k}$.

On the foundation of above definitions and reasoning, the generation algorithm of connective part units is listed as follows:

Generation flow of connective part units is showed in Figure 1. 


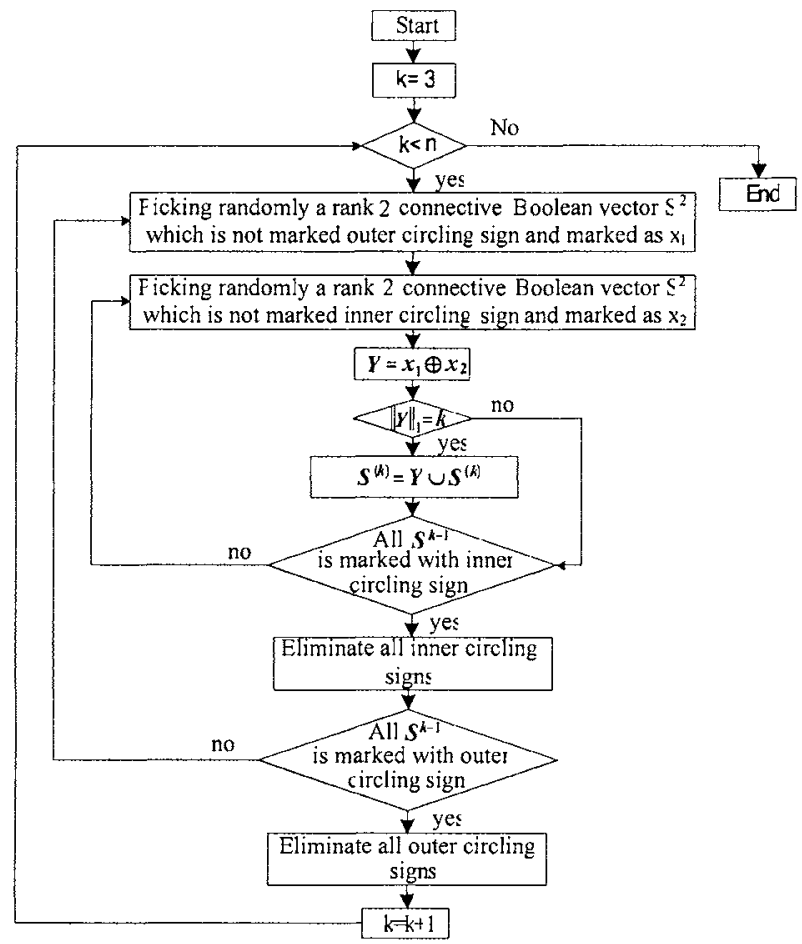

Fig. 1. Generation flow chart of connective part units

Step1: Let $\mathrm{k}=3$.

Step2: To pick randomly a 2-rank connective Boolean vector $\mathrm{S}^{2}$ which is not marked outer circling sign, and mark it as $\mathrm{x}_{1}$.

Step3: To pick randomly a 2-rank connective Boolean vector $S^{2}$ which is not marked inner circling sign, and mark it as $\mathrm{x}_{2}$.

Step4: To calculate Boolean sum of $x_{1}$ and $x_{2}$, that is $x_{1} \oplus x_{2}$. And let it equal $Y$.

Step5: To determine whether $\mathrm{Y}$ is 3-rank Boolean vector or not. If yes, then $S^{k}=S^{k} \cup Y$. Or turn to step6.

Step6: To determine whether there are still 2-rank Boolean vectors which are not marked with inner circling signs. If yes, turn to step3. Or cancel all 2-rank Boolean inner signs and turn to step7.

Step7: To determine whether there are still 2-rank Boolean vectors which are not marked with outer circling signs. If yes, turn to step2. Or to eliminate all outer circling signs of 2-rank Boolean vectors, then turn to step8.

Step8: To determine whether $k+1$ is smaller than $n$. If yes, let $k=k+1$. Or turn to end. 


\section{Disassembling Model}

For a connective part unit, the process of determining whether it can generate part units on next lower level by taking down one part from it is called disassembling determining. This paper advances a kind of possible displacement model of part and uses possible displacement equation groups as disassembling sieve to eliminate illogical part units from connective part units set.

\subsection{Disassembling model}

Generally, the premise of disassembly of a part is that there should be no any blockage on its disassembly channel. That is to be sure that disassembly channel exits. Possible displacement model describes blockage relation in disassembly in the direction $\pm \mathrm{X}, \pm \mathrm{Y}, \pm \mathrm{Z}$. Logical equation which is constituted with a group of parts that block the disassembly channel of some part is called possible displacement equation of the part [14]. Possible displacement equation expresses channel blockage relation in disassembly. For example, for part $a_{k}$, if its disassembly channel is blocked, then $W\left(a_{\mathrm{k}}\right)=1$. On the contrary, $W\left(a_{\mathrm{k}}\right)=0$. For the part $a_{\mathrm{j}}$ which is a element constituting possible displacement equation of $a_{\mathrm{k}}$, if it is disassembled before $a_{\mathrm{k}}$, then its logical value in equation $W\left(a_{\mathrm{k}}\right)$ is $a_{j}=0$. On the contrary, $a_{\mathrm{j}}=1$. Logic relation between each two parts in possible displacement equation is AND/OR. Possible displacement equations of all parts constitute possible displacement model of product.

Table 1 below shows possible displacement model where $\left(a_{i}, a_{k}\right)$ denotes logical relation of $a_{\mathrm{k}}$ to $a_{\mathrm{j}}, F_{1}-F_{6}$ denotes blockage relation in the direction $\pm \mathrm{X}, \pm \mathrm{Y}, \pm \mathrm{Z}$ and $\bullet$ denotes blockage of channel.

Table 1. Possible displacement model

\begin{tabular}{ccccccc}
\hline & $\mathrm{F} 1$ & $\mathrm{~F} 2$ & $\mathrm{F3}$ & $\mathrm{F} 4$ & $\mathrm{F5}$ & $\mathrm{F6}$ \\
\hline$\left(\mathrm{a}_{1}, \mathrm{a}_{2}\right)$ & $\cdot$ & & & & $\cdot$ \\
$\ldots$ & & & & & & \\
$\left(\mathrm{a}_{3}, \mathrm{a}_{4}\right)$ & & & & & \\
$\ldots$ & & & & & \\
$\left(\mathrm{a}_{5}, \mathrm{a}_{7}\right)$ & & & & & \\
$\ldots$ & & & & \\
$\ldots$ & & & & & \\
$\left(\mathrm{a}_{11-1}, \mathrm{a}_{11}\right)$ & $\cdot$ & & & & & \\
\hline
\end{tabular}




\subsection{Obtaining algorithms of possible displacement equation groups}

To obtain possible displacement equation groups from possible displacement model, below definitions are given:

(1) $F_{1}-F_{6}$ shows blockage relation of movement in the direction $\pm \mathrm{X}, \pm \mathrm{Y}, \pm \mathrm{Z}$.

(2) $F_{\mathrm{i}}\left(a_{\mathrm{k}}, a_{\mathrm{j}}\right)$ show the blockage relation of $a_{\mathrm{j}}$ to $a_{\mathrm{k}}$ where $1 \leq i \leq 6,1 \leq k, j \leq n$, and $j \neq k$ ( $\mathrm{n}$ is the total number of parts).

(3) Possible displacement relation of $a_{\mathrm{j}}$ to $a_{\mathrm{k}}$ is different from $a_{\mathrm{k}}$ to $a_{\mathrm{j}}$. That is $F_{\mathrm{i}}\left(a_{\mathrm{k}}, a_{\mathrm{j}}\right) \neq F_{\mathrm{i}}\left(a_{\mathrm{j}}, a_{\mathrm{k}}\right)$. Obviously, $F_{\mathrm{l}}\left(a_{\mathrm{k}}, a_{\mathrm{j}}\right)=F_{2}\left(a_{\mathrm{j}}, a_{\mathrm{k}}\right), F_{3}\left(a_{\mathrm{k}}, a_{\mathrm{j}}\right)=F_{4}\left(a_{\mathrm{j}}, a_{\mathrm{k}}\right)$ and $F_{5}\left(a_{\mathrm{k}}, a_{\mathrm{j}}\right)=$ $F_{6}\left(a_{\mathrm{j}}, a_{\mathrm{k}}\right)$.

Algorithm of obtaining possible displacement groups is as follows:

(1) To extract possible displacement information from possible displacement model in table 1 .

(2) Obtaining steps:

Step1: To determine whether part $a_{\mathrm{k}}$ is interfered.

$W\left(a_{k}\right)=\bigvee_{j=1}^{n}\left[\bigvee_{i=1}^{6} F_{i}\left(a_{k}, a_{j}\right)\right]$, if $W\left(a_{k}\right)=0$, then it means that part $a_{k}$ is not interfered. Or if $W\left(a_{k}\right)=1$, then part $a_{k}$ is interfered.

Step2: To obtain combination of parts that interfere $a_{k}$.

If $\bigvee_{i=1}^{6}\left[F_{i}\left(a_{k}, a_{j}\right) \vee F_{i}\left(a_{k}, a_{m}\right)\right]=1$, then $W\left(a_{k}\right)=a_{j} \wedge a_{m}$

Step3: Combinatorial law of polychromatic sets: if $W\left(a_{k}\right)=a_{j} \wedge a_{m}, W\left(a_{k}\right)=a_{j} \wedge$ $a_{l}$. Then $W\left(a_{k}\right)=a_{j} \wedge\left(a_{m} \vee a_{l}\right)$.

\subsection{Generation algorithm of disassembly part unit}

Determining disassembling of all connective part units by using possible displacement equation groups, those part units that are determined as disassembling ones are called sub-part units, generation algorithm of which is as follows:

Figure 2 shows generation flow of part unit. 


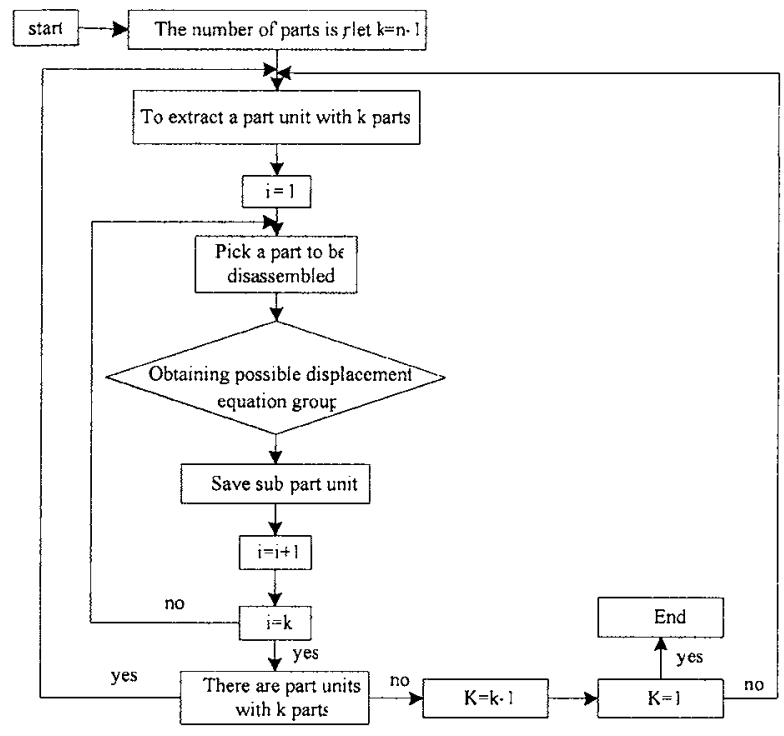

Fig. 2. Flow chart of generation of disassembly unit

Step1: To extract all part combinations with n-1 parts and pick out those combinations that meet possible displacement groups, they are sub-part units with n1 parts and save them to unit database.

Step2: To pick a part unit composed of $k$ parts.

Step3: To pick a part from $k$ parts of the part unit of step 2 as the disassembled one.

Step4: To determine whether every part to be disassembled meets its possible displacement equation groups. If yes, then the disassembly is proper and save the part unit with k-1 parts to sub-part unit database.

Step5: To determine whether every part has been selected as disassembled one. If yes, then turn to step6. Or turn to step3.

Step6: To determine whether there are part units with $\mathrm{k}$ parts. If yes, then turn to step2. Or let $\mathrm{k}=\mathrm{k}-1$.

Step7: To determine whether $k$ is smaller than 1. If yes, then stop. Or turn to step2.

\section{Generation Method of Disassembly Sequence}

On the basis of obtaining possible displacement model, generation method of disassembly sequence is detailed as follows. Computer reads in possible displacement model and saves it in matrix, then searches every line of the matrix and calculates cumulative constrains of each part. Disassembly sequence can be 
generated according to the principle that part which has less cumulative constrains be disassembled firstly.

\section{Disassembly Hierarchical Graph}

After obtaining sub-part unit, we employ hierarchical graph of polychromatic sets to express level relation in disassembly [15], where set of vertexes is:

$$
A=\left(A, A_{1}^{\prime \prime}, A_{2}^{\prime \prime}, \cdots, A_{k}^{\| \prime}, A_{l}^{\prime \prime \prime}, \cdots, A_{m}^{N}, a_{1}, a_{2}, \cdots a_{n}\right)
$$

Border $\mathrm{e}_{\mathrm{i}, \mathrm{j}} \in$ connects node $A_{\mathrm{j}}{ }^{\mathrm{J}}$ and node $A_{\mathrm{j}}{ }^{\mathrm{j}+1}$ on the next lower level. Borders in graph $G=(A, E)$ are composed as follows:

$$
E \ni e_{i(j)}=\left\{\begin{array}{l}
1, \text { if } A_{\mathrm{i}}^{\mathrm{J}} \ni A_{\mathrm{j}}^{\mathrm{J}+1} \\
0, \text { on the contrary }
\end{array}\right.
$$

Steps of building hierarchical graph are as follows:

Step1: Root node of the hierarchical graph is the set of all parts of product.

Step2: Beginning from root node, nodes on every level can be decompounded into one disassembly unit on next lower level and a single part. The disassembly unit can be decompounded once again. For example, node of disassembly unit in rank $J$ can be decompounded into the elements of rank $(J+1)$ including the disassembly unit of $A_{\mathrm{J}+1}$ and the part of $a_{\mathrm{k}} . A_{\mathrm{J}+1}$ and $a_{\mathrm{k}}$ meet the condition of $A_{\mathrm{J}+1} \cup a_{\mathrm{k}}=A_{\mathrm{J}}, \mathrm{k}<\mathrm{n}$, and $A_{\mathrm{J}+1}$ can be divided once again.

Step3: Similarly, the hierarchical graph has been built when every disassembly unit has been decompounded into one single part, and then the disassembly sequence of product can be obtained from the hierarchy graph expediently.

\section{Case Analyzing}

Take PC box as an example to show application of present disassembly model in three-dimension disassembly. In Figure 3 each number denotes separately as follows. 1-box, 2-graphical card, 3-audio card, 4-motherboard, 5-speaker, 6-harddisk, 7-soft driver, 8-shelf, 9-power.
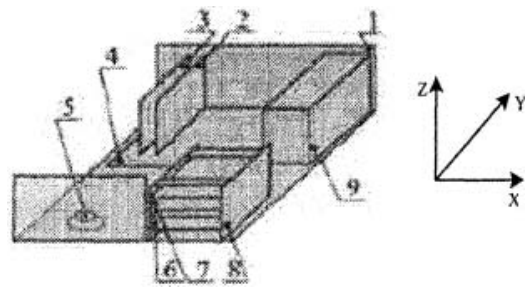

Fig. 3. Sketch map of PC box

(1) Connective part units 
Total number of part units with $\mathrm{n}$ parts is $\left(2^{n}-n-2\right)$. For this example, $\mathrm{n}$ is 9 , so total number of part units is 501 . Number of connective part units is calculated as 164 .

(2) Possible displacement model

Possible displacement model of PC box is showed in Table 2. And possible displacement equation groups are as follows:

$$
\begin{gathered}
W\left(a_{1}\right)=a_{4} \vee a_{5} \vee a_{8}, W\left(a_{2}\right)=0, W\left(a_{3}\right)=0 W\left(a_{4}\right)=a_{2} \vee a_{3} \vee a_{7}, \\
W\left(a_{5}\right)=0, W\left(a_{6}\right)=a_{7}, W\left(a_{7}\right)=0, W\left(a_{8}\right)=a_{6} \vee a_{7}, W\left(a_{9}\right)=0 .
\end{gathered}
$$

\begin{tabular}{|c|c|c|c|c|c|c|}
\hline & F1 & $\mathrm{F} 2$ & $\mathrm{~F} 3$ & $\mathrm{~F} 4$ & F5 & F6 \\
\hline$\left(a_{1}, a_{2}\right)$ & & & & - & - & \\
\hline$\left(a_{1}, a_{3}\right)$ & & & & • & • & \\
\hline$\left(a_{1}, a_{4}\right)$ & & & & • & • & \\
\hline$\left(a_{1}, a_{5}\right)$ & & & & & - & • \\
\hline$\left(a_{1}, a_{6}\right)$ & & & & & • & \\
\hline$\left(a_{1}, a_{7}\right)$ & & & & & • & \\
\hline$\left(a_{1}, a_{8}\right)$ & & & • & & • & \\
\hline$\left(a_{1}, a_{9}\right)$ & & & & - & - & \\
\hline$\left(a_{2}, a_{4}\right)$ & • & • & • & • & & • \\
\hline$\left(a_{3}, a_{4}\right)$ & • & • & • & • & & • \\
\hline$\left(a_{4}, a_{9}\right)$ & & & & & • & \\
\hline$\left(a_{6}, a_{7}\right)$ & & & & & • & \\
\hline$\left(a_{1}, a_{2}\right)$ & • & • & • & & • & - \\
\hline$\left(a_{7}, a_{8}\right)$ & - & - & • & & - & - \\
\hline
\end{tabular}

Table 2. Possible displacement model of PC box

(3) Obtaining of sub disassembly unit

By using generation algorithms of disassembly unit, number of part units on every level is calculated as following:

$$
\begin{aligned}
& \quad\left|A^{(8)}\right|=5,\left|A^{(7)}\right|=11,\left|A^{(6)}\right|=14,\left|A^{(5)}\right|=13,\left|A^{(4)}\right|=13,\left|A^{(3)}\right|=7, \\
& \left|A^{(2)}\right|=3 .
\end{aligned}
$$

(4) Disassembly sequence

Combining possible displacement model above and method in section 3 , constrain number of every part is calculated as:

$$
\begin{aligned}
& R\left(a_{1}\right)=13, R\left(a_{2}\right)=6, R\left(a_{3}\right)=6, R\left(a_{4}\right)=8, R\left(a_{5}\right)=2, R\left(a_{6}\right)=7, \\
& R\left(a_{7}\right)=7, R\left(a_{8}\right)=12, R\left(a_{9}\right)=3 .
\end{aligned}
$$

According to order of constrain number above, we can get the disassembly sequences as following:

$$
\begin{aligned}
& \left(a_{5} \rightarrow a_{9} \rightarrow a_{2} \rightarrow a_{3} \rightarrow a_{6} \rightarrow a_{7} \rightarrow a_{4} \rightarrow a_{8} \rightarrow a_{1}\right) \\
& \left(a_{5} \rightarrow a_{9} \rightarrow a_{3} \rightarrow a_{2} \rightarrow a_{6} \rightarrow a_{7} \rightarrow a_{4} \rightarrow a_{8} \rightarrow a_{1}\right)
\end{aligned}
$$




$$
\begin{aligned}
& \left(a_{5} \rightarrow a_{9} \rightarrow a_{2} \rightarrow a_{3} \rightarrow a_{7} \rightarrow a_{6} \rightarrow a_{4} \rightarrow a_{8} \rightarrow a_{1}\right) \\
& \left(a_{5} \rightarrow a_{9} \rightarrow a_{3} \rightarrow a_{2} \rightarrow a_{7} \rightarrow a_{6} \rightarrow a_{4} \rightarrow a_{8} \rightarrow a_{1}\right)
\end{aligned}
$$

(5) Hierarchical graph

Hierarchical graph is built on the foundation of four disassembly sequences above. They are illustrated as follows (a), (b), (c) and (d) in Figure 4.

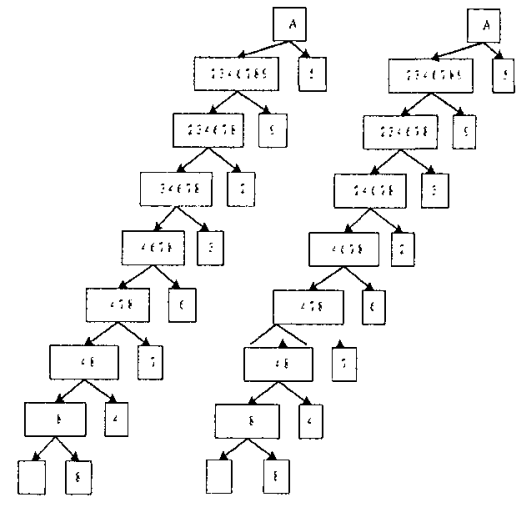

(a) (b)

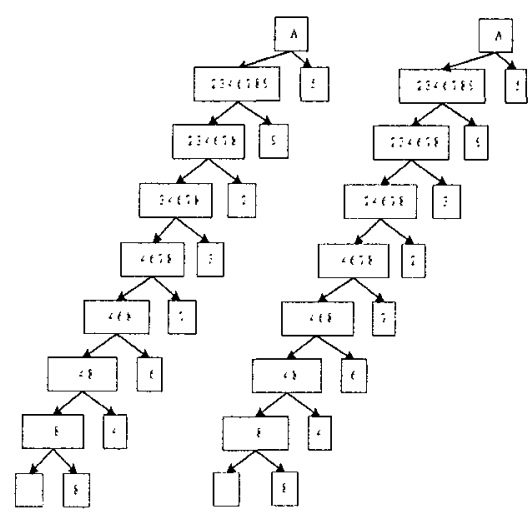

(c)

(d)

Fig. 4. Hierarchical graph

\section{Conclusions}

This paper presents concept of connectivity of part units and their generation algorithms. Connective part units are generated directly by using the generation algorithms of connective part units in order to avoid disturbing of many disconnection part units. Connective part units are sieved by possible displacement equation groups which are based on possible displacement model to eliminate illogical part units. It is effective to defend combinatorial explosion of disassembly schemes. Based on case, disassembly sequences are programmed according to the principle that part which has less cumulative constrains should be disassembled firstly, and the disassembly sequences which are consistent with real process of disassembly are obtained. Finally the process of disassembly is expressed by building hierarchical graph of polychromatic sets. This expression is concise and intuitionistic. Polychromatic sets theory adopts logical Boolean operation. It has following advantages: complexity of algorithms is less, easy to program and facilitate to extend to complex system. 


\section{References}

1. P. Veerakamolmal and S. Gupta, A Case Based Reasoning Approach For Automating Disassembly Process Planning, Journal of Intelligent Manufacturing 13(1), 47-60 (2002).

2. V. D. R. Guide Jr., V. Jayareman, and R. Srivastava, The Effect of Lead-Time Variation on the Performance of Disassembly Release Mechanisms, Computers \& Industry Engineering 36(4), 759-779 (1999).

3. K.E. Moore, A. Gunger, and S.M. Gupta, A Petri Net Approach to Disassembly Process Planning, Computer and Industrial Engineering 35(1), 165-168 (1998).

4. A. Gungor and S. M. Gupta, Disassembly Sequence Planning for Products with Defective Parts in Product Recovery, Computer and Industrial Engineering 35(1), 161-164 (1998).

5. A.J.D. Lambert, Determining Optimum Disassembly Sequences in Electric Equipment, Computers \& Industry Engineering 43(3), 553-575 (2002).

6. E. Kongar and S.M. Gupta, A Genetic Algorithm for Disassembly Process Planning, In the Proceedings Of Environmentally Conscious Manufacturing II, edited by S.M. Gupta (The international Society for Optical Engineering, Newton, MA, united state, 2002), pp. 54-62.

7. H-S Park, H-W Choi, and H-S.Mok, Disassembly Sequence Planning for an End-of-Life Car, In the Proceedings of 7th Korea-Russia International Symposium on Science and Technology, edited by J.H. Lee (Univ. Ulsan, Ulsan, south Korea, 2003), pp. 299-304.

8. S.M. Gupta, E. Erbis, and S.M. Mcgovern, Disassembly Sequencing Problem: A Case Study of a Cell Phone, In the Proceedings of Environmentally Conscious Manufacturing IV, edited by S.M. Gupta (International Society for Optical Engineering, Philadelphia, PA, United State, 2004) pp. 43-52.

9. B. Gonzalez and B. Adenso-diaz, A Scatter Search Approach to the Optimum Disassembly Sequence Problem, Computer \& Operation Research 33(6), 1776-1793 (2004).

10. C. Chung and Q. Peng, An Integrated Approach to Selective-Disassembly Sequence Planning, Robotics and Computer-Integrated Manufacturing 21(4-5), 475-485 (2005).

11. G. Villalba, M. Segarra, J.M. Chimenos, and F. Espiell, Using the Recyclability Index of Materials as a Tool for Design For Disassembly, Ecological Economics 50(3-4), 195-200 (2004).

12. J-F Li, J. Chen, F-Y Li, and Y-L Wang, Disassembly and Recycling Model of MachElectricity Products and Generation of Disassembly Sequence, Transaction of ShanDong University(industry edition) 35(5), 38-40 (2004).

13. J. Gao, D. Xiang, and H. Chen, Connectivity Sieve in Disassembly AND/OR Model, Transaction of Tsinghua University 43(8), 1046-1048 (2003).

14. V.V. Pavlov, Z. Li, and W. Dai, Simulation of Assembly Sequence Based on Polychromatic Sets, Transaction of Xi'an Jiaotong University 35(11), 1208-1210 (2001).

15. Z. Li, Research and Application of Polychromatic Sets in Advanced Manufacturing (China WaterPower Press and Knowledge Press, Beijing, 2005). 